\author{
Gad Alon* \\ Associate Professor, University of Maryland, School \\ of Medicine, Department of Physical Therapy \& \\ rehabilitation Sciences, 100 Penn Street, Baltimore, \\ MD 21201 \\ Dates: Received: 29 June, 2014; Accepted: 20 \\ August, 2014; Published: 22 August, 2014 \\ *Corresponding author: Gad Alon Ph.D., PT, \\ Associate Professor, University of Maryland, School \\ of Medicine, Department of Physical Therapy \& \\ rehabilitation Sciences, 100 Penn Street, Baltimore, MD \\ 21201, E-mail: galon@som.umaryland.edu \\ www.peertechz.com \\ ISSN: 2455-5487 \\ Keywords: Gender; Upper extremity; Stroke; \\ Function; Loss
}

\section{Research Article \\ Loss of upper Extremity Motor Control and Function affect Women more than Men}

\begin{abstract}
Background: Loss of functional ability and motor control following stroke appears to affect women more severely than men in general. However, little attention has been paid specifically to the upper extremity.

Objective: To quantify loss of upper extremity control, comparing men to women that survived ischemic stroke. A secondary purpose was to report gender differences in residual deficits $(\mathrm{RD})$ of the paretic upper extremity following 12 weeks of rehabilitation.

Design: A retrospective data analyses from women $(n=21)$ and men $(n=24)$ that received task-specific (control) or task-specific + functional electrical stimulation (FES) training to the paretic upper extremity.

Methods: Participants performed a modified Fugl-Meyer (mF-M), the Box \& Blocks and the Jebsen-Taylor light object lift tests. Baseline and post 12-week training data of women and men were compared statistically $(p=0.05)$. RD was calculated as $=(1-($ paretic $/$ non-paretic) $) * 100$.

Results: Females had significantly greater loss of upper extremity control compared to male subjects $(9.3 \pm 8.6$ vs. $14.8 \pm 12.0 \mathrm{mF}-\mathrm{M}$ score; $0.7 \pm 1.9$ vs. $4.5 \pm 6.6$ transferred blocks; and $60 \pm 0$ vs. $49.9 \pm 18.3 \mathrm{sec}$ completing the Jebsen-Taylor test). Females' RD were significantly higher performing the $\mathrm{mF}-\mathrm{M}$ and Box \& Blocks than the males' RD. The Jebsen-Taylor test's RD did not differ statistically between the genders.

Limitations: The study was not based on prospective analysis of data where gender is considered a factor in the original experimental design.

Conclusion: Loss of upper extremity control is considerably more evident in females following first-time ischemic stroke. The recovery rate associated with task-specific rehabilitation with or without FES appears similar in a sub-group with good prognosis. The deficits of motor control and hand function of females with poor prognosis remain significantly higher than the deficits of males with similar prognosis.
\end{abstract}

\section{Background}

Loss of functional ability and motor control following ischemic or hemorrhagic stroke is a devastating event that appears to affect women more severely than men worldwide [1-5]. However, until recently, the details and meaning of such a global statement has not been explored. Little attention has been paid specifically to the upper extremity, despite a clear indication that only 12 percent of patients with a first time stroke are expected to fully recover upper extremity function [6]. Moreover, for the majority of stroke survivors the inability to use the paretic hand also means that bilateral upper extremity daily functions are no longer a viable option $[7,8]$.

Wolf and colleagues [3] were among the first to document the differential effects of gender on upper extremity performance. Using the log mean Wolf Motor Function Test (lmWMFT) they reported that female subjects were slower than men performing the test.
However, the relevance of these findings was limited to that specific test. More importantly, these patients had a stroke 3-9 months earlier, already recovered, or never lost measurable volitional motor control of the shoulder, elbow, wrist and fingers.

The vast majority of patients, having paralysis or paresis of the upper extremity, are not likely to regain control of all the movement components described by Wolf et al. [9] Furthermore, studies that focused on recovery of the upper extremity following a stroke frequently used different outcome measures [10-15]. In relation to upper extremity function, the Action Research Arm Test (ARAT), the Jebsen-Taylor Hand Function test (J-T) and the Box \& Blocks $(B \& B)$ are frequently used by different groups of investigators $[6,10$ 12,14-16]. One conceivable advantage of these tests, particularly the $\mathrm{J}-\mathrm{T}$ and $\mathrm{B} \& \mathrm{~B}$ is the less time intensive administration and data compilation process. In addition both can be done at any location including patients' homes, and the two tests are complementary to 
each other. The $\mathrm{B} \& \mathrm{~B}$ tests the ability to grasp, move, and release small objects close to the body [17], and two tasks of the J-T test battery require full opening of the hand, grasping, lifting light or heavy objects, and placing them away from the body $[12,18]$. The two tests appear applicable to daily use of the paretic upper extremity, but are not relevant to bimanual daily functions $[7,8]$.

The test most frequently reported to assess loss of upper extremity motor control following stroke is the Fugl-Meyer (F-M) $[6,9,11,15,19]$ or the recently modified Fugl-Meyer (mF-M) [14,20,21]. However, the severity of upper extremity loss whether the outcome measure is functional or motor control, has not been documented for women and men separately. Such documentation would be important particularly because the severity of upper extremity paralysis/paresis appears to be the strongest predictor of recovery $[6,22]$. For example, Kwakkel et al. provided a prediction model based on data from 102 new survivors of stroke suggesting that having upper extremity Fugl-Meyer scores of 11 points in the second week post-stroke, increasing to 19 points in the fourth week, would predict with $83 \pm 8 \%$ certainty the recovery of some dexterity at 6 months [6]. Similarly, Lang and Beebe [13] constructed a model based on 3-d measurements of active range of motion (AROM) and studied 32 chronic strokes. AROM predicted $73 \%$ of the variance in hand function. But neither model considered gender as a factor, raising the fundamental question of the sensitivity of the model if women's loss and recovery of mF-M score or AROM turned out to be different than men's. Before answering this question the need to establish gender differences in loss of motor control and functional ability of the paretic upper extremity shortly after stroke should be documented. Thus, the primary purpose of this study was to quantify upper extremity loss of motor control and functional ability, comparing men to women that survived a recent (1-4 weeks) ischemic stroke. A secondary purpose was to report gender differences in residual deficits (RD) of the paretic upper extremity following 12 weeks of rehabilitation. The definition, validation, and potential utility of $\mathrm{RD}$ as an outcome measure in stroke rehabilitation have been recently published [23].

\section{Method}

\section{Subjects}

The methodology and original data comparing the effects of taskspecific only versus task-specific + functional electrical stimulation (FES) training on the recovery of the paretic upper extremity, shortly after an ischemic stroke, have been published [14-21]. Inclusion and exclusion criteria are listed in table 1 . This study includes four additional subjects not available in the previous publications and represent a retrospective data analyses of women $(n=21)$ and men $(n=24)$ that completed the above studies. The demographic profiles and training group assignments are summarized in table 2 . There were no statistical differences between groups regarding age, time elapsed between stroke onset and admission to a rehabilitation center, admission to start of study, or Folstein mini mental score.

\section{Measurements}

Functional assessments focused on the Box \& Block (B\&B) and the light object lift sub-set of the Jebsen-Taylor (J-T) tests. These tests are commonly reported, yet they are not time or burden-

\section{Table 1: Inclusion/exclusion criteria}

\section{Inclusion}

1. Single unilateral ischemic stroke that occurred no fewer than 2 weeks and no more than 4 weeks before study commencement (ensuring that patients are medically stable)

2. Paralysis/paresis of the upper limb

3. Fugl-Meyer score between 2 and 40

4. No clinical evidence of limited passive range of motion of the paralyzed upper limb

5. Age 20-90 years

6. Admitted to Kernan Hospital for at least 1 week and actively engaged in physical therapy, occupational therapy or both

7. Forearm and hand size compatible with the use of the $\mathrm{H}-200$ stimulation system

8. At least $60 \%$ of full finger flexion and extension response to stimulation

9. Able and willing to participate in a 12-week study

10. Adequate language function to understand and respond appropriately to two-step commands (determined by the evaluation to sign a consent form questionnaire)

11. Ability and willingness to sign the consent form approved by the Institutional review board (IRB)

\section{Exclusion}

1. Implanted electronic pacing or defibrillation device, unstable vital signs or potentially fatal cardiac arrhythmia

2. Active reflex sympathetic dystrophy, shoulder-hand syndrome, or other existing residual weakness attributed to lower motor neuron lesions of either upper limb

3. Inability to sit in a standard armless chair for 30 min (needed for testing)

4. Patients with sensory (receptive) aphasia, or impaired communication that lead to an inability to understand the study procedures

5. Any comorbid neurological disease or condition such as multiple sclerosis, Parkinson's disease, spinal cord injury, traumatic brain lesion, or brain tumor

6. Clinical evidence of shoulder subluxation (one finger or greater separation)

7. Caregiver unavailable to help with the training protocol, in a situation of patient dependency

8. Refusal to be videotaped (videotape was a critical component of the testing)

9. Mini Mental Status Examination score of 21 or lower

intensive functional tests. A video-based modified Fugl-Meyer score $(\mathrm{mF}-\mathrm{M})$ for the upper extremity was used to measure motor control loss and recovery [20]. Due to low tolerance for lengthy testing and considerable paralysis of the upper extremity during inpatient rehabilitation, testing was limited to 30 -minute sessions. Data were obtained from both paretic and non-paretic upper extremities. The three outcome measures were recorded at baseline, and after 4,8 , and 12 weeks of training.

Modified Fugl-Meyer test $(\mathrm{mF}-\mathrm{M})$ : The patient sat on a standard armless chair (seat height $46 \mathrm{~cm}$ ) facing the video camera (frontal view) and was instructed to move her/his upper extremity in accordance with the F-M movement items. For movements that were better observed from the sagittal view the patient was turned 90 degrees to the camera's lens. A total of 27 movement items were scored as follows: no visible movement $=0$; partial movement $=1$; and full range movement $=2$. The modified version did not include the original items A (reflex activity) and item D (coordination/speed) [20]. Consequently the maximum score was 54 points.

Box \& Blocks Test (B\&B): This test included a commercially available box divided by a partition and containing 150 (2.54 x 2.54 $\mathrm{cm}$ ) blocks located on one side. The box was placed facing the patient, 
Table 2: Patients' characteristics at study onset.

\begin{tabular}{|l|c|c|c|c|}
\hline & Age (Yrs) & Stroke to Study Onset (Days) & Folstein Mini mental Score & Prognosis Poor/Good \\
\hline Females & $62.6 \pm 15.6$ & $20.3 \pm 10.2$ & $27 \pm 2.7$ & 12 vs. 9 \\
\hline Males & $61.5 \pm 12.9$ & $17.3 \pm 7.8$ & $26.6 \pm 2.4$ & $9 / 21$ \\
\hline
\end{tabular}

Data presented as groups mean \pm standard deviation. Statistically, females and males data did not differ significantly ( $p>0.1$ ).

$13 \mathrm{~cm}$ back from the edge of a $76 \mathrm{~cm}$ high desk. The box's partition bisected the midline of the subject's body. Patients had to pick up one block at a time and transfer it to the other side of the box as fast as possible. Upon command to start, the subjects began transferring the blocks. At the end of 60 seconds, the transferred blocks were counted. The test was repeated 3 times with each hand, and the highest scores achieved (one paretic and one non-paretic) were included as the final outcome measure.

Jebsen-Taylor Light Object Lift Test (J-T): The J-T test evaluated the ability to open the hand, grasp, hold, move and place a large objects away from the body. The patient sat in a standard environment (seat height $46 \mathrm{~cm}$, desk height $76 \mathrm{~cm}$,) facing 5 empty aluminum cans (11 $\mathrm{cm}$ in height, $8 \mathrm{~cm}$ in diameter, and weighing 57 gram) placed in a row, $5 \mathrm{~cm}$ apart in front of a board. The board was secured to the desk $13 \mathrm{~cm}$ from its front edge. Upon command to begin, the patient grasped a can, lifted it over a $5 \mathrm{~cm}$ vertical barrier, and placed it back on the board on the other side of the barrier. The time (in sec) it took to move all 5 cans was measured using a stopwatch. Patients who were unable to perform the test or did not complete the test in $60 \mathrm{sec}$ received $60 \mathrm{sec}$ as their score. The test was repeated 3 times with each hand, and the fastest time recorded for each hand was used as the final outcome measure.

\section{Training procedure}

Two separate studies, detailing the task-specific or the taskspecific plus FES training programs have been published. One included females and males with good prognosis ${ }^{14}$ and one including both genders with poor prognosis [21]. Patients with good prognosis (11 males and 9 females) began training with active-assistive exercises. With further recovery of motor control, participants were progressed to practice task-specific activities including grasping and moving objects and using the upper extremity in routine activities of daily living. Out of these 20 subjects, 4 males and 5 females were assigned to a task-specific + FES and followed the same individual task-specific training program the task-specific without FES ( 7 males and 4 females) followed. Patients using the FES practiced for 30 min sessions, twice each day and combined the exercises with an electrical stimulation program. This FES program stimulated the wrist/finger flexors and extensors in a manner that induced opening and closing of the paretic hand. After completing each $30 \mathrm{~min}$ period of the combined FES and task-specific exercises, the patients continued to have the stimulation artificially open and close the hand for an average of an additional 2 hours per day, but without practicing specific exercises. The FES system used in the study was the $\mathrm{H}-200^{\mathrm{TM}}$ (Bioness, Inc.) and has been described in previous publications $[10,14,21]$.

Patients with poor prognosis typically had minimal or no volitional movements in the upper extremity. Accordingly, they began with very simple and predominantly passive exercises and, as motor recovery took place, progressed to active assistive exercises and then to active, task-specific exercises (mostly grasping, holding, moving and placing objects toward and away from the body). In this group, 7 out of 13 males, and 4 out of 12 females were randomly assigned to practice task-specific $+\mathrm{FES}$ and used the same protocol as the patients with good prognosis.

All patients practiced their individually-tailored program with the attending therapists ( $30 \mathrm{~min}$ sessions, given twice daily) five days each week during hospitalization; after discharge they were instructed to practice 30 minutes twice a day without supervision. In addition, all patients continued to receive in-home physical and occupational therapy 1-2 times per week through their standard medical coverage. The research therapist visited each patient once a week and modified the research-related exercise program based on patient progress. The actual amount of time that each patient exercised at home was not monitored due to budget constraints. Therefore, the degree of compliance was unknown. Patients reported they were doing the exercises and FES at home, but not always as instructed.

\section{Data management}

Because the added value of FES to task-specific training has been published $[14,21]$ this retrospective analysis focused only on the gender factor. Data from the modified Fugl-Meyer (mF-M), the Box \& Blocks and the Jebsen-Taylor light object lift tests were analyzed at two time points: one at study onset just prior to the beginning of training, and the second after 12 weeks of training. Data of women versus men were compared using independent t-test or Mann-Whitney U-test for normally and non-normally distributed data respectively $(\mathrm{p}=0.05)$. Residual deficits (RD) of the paretic upper extremity at the end of intervention was calculated as $\mathrm{RD}=(1-(\text { paretic/non-paretic }))^{\star} 100$ [23].

\section{Results}

Males (17.3 \pm 7.8 days post-stroke), and females (20.3 \pm 10.2 days post-stroke) exhibited considerable inter-subject variability in the magnitude of motor and functional loss in all three baseline outcome measures. The females' $\mathrm{mF}-\mathrm{M}$ score was only $9.3 \pm 8$.6 points compare to males' score of $14.8 \pm 12.0$ points. The difference between gender did not reach statistical significance $(\mathrm{p}=0.08)$. In the two functional tests the gender difference was more evident. Only 3 of 21 females were able to transfer at least one block having a group mean of $0.7 \pm 1.9$ blocks. This contrasted with 10 of 24 males whose group's mean was $4.5 \pm 6.6$ blocks; a statistically significant better performance $(\mathrm{p}=0.02)$. Similarly, no female participants were able to complete the J-T test in less than $60 \mathrm{sec}$; while 6 of 24 males completed it successfully. Group differences were statistically significant $(\mathrm{p}=0.01)$.

Residual deficits (RD) after 12 weeks of training remained substantial. Females $\mathrm{mF}-\mathrm{M}$ deficits were $53.2 \pm 34 \%$ compared to males deficits at $32 \pm 25.1 \%(\mathrm{p}=0.02)$. Remaining deficits in transferring 
blocks with the paretic upper extremity were $75 \pm 32.2 \%$ and $51.4 \pm 40.4 \%$ for females and males respectively $(\mathrm{p}=0.02)$. Individual improvement in completing the J-T light object lift test after 12 weeks training was evident; as 18 of 24 males and 10 of 21 females successfully completed the task. Nonetheless, males were $6.1 \pm 6.6$ times slower transferring the 5 cans with the paretic hand compared to the non-paretic hand; while females were $8.1 \pm 5.9$ time slower with their paretic hand. However, the difference between genders was not significant $(\mathrm{p}=0.35)$

Further qualitative probing of the data revealed that clustering patients into two subgroups based on severity of paralysis resulted in 25 patients ( 13 females and 12 males) having severe paralysis and an initial mF-M score of 2-10 while 20 patients ( 8 females and 12 males) retained some volitional movement and had an initial $\mathrm{mF}-\mathrm{M}$ score between 11 and 33 . Figure 1 illustrates the changes in $\mathrm{mF}-\mathrm{M}$ score over 12 weeks. As seen, females with the most severe paralysis gained numerically and statistically $(\mathrm{p}=0.004)$ the least amount of motor control; compared to males with the same initial level in severity of paralysis. In contrast, females in the cluster of patients having an initial mF-M between 11 and 33 and therefore are in line with Kwakkel et al. prognostic model for good recovery [6], scored lower initially but recovered to the same level of males at study end point.

\section{Discussion}

This retrospective data analyses provide a new clinical insight describing the loss and recovery of upper extremity motor control and hand function status post an ischemic stroke. The findings that one measure of motor control loss (mF-M) and two assessments of upper extremity functional deficit (B\&B and $\mathrm{J}-\mathrm{T}$ sub test) affected women more than men add robustness to the knowledge that physical rehabilitation following stroke should consider gender an important factor. In our sample, 7 of 24 males (29\%) but only 3 of 21 females (14\%) recovered $90 \%$ or above the $\mathrm{mF}-\mathrm{M}$ maximal score.

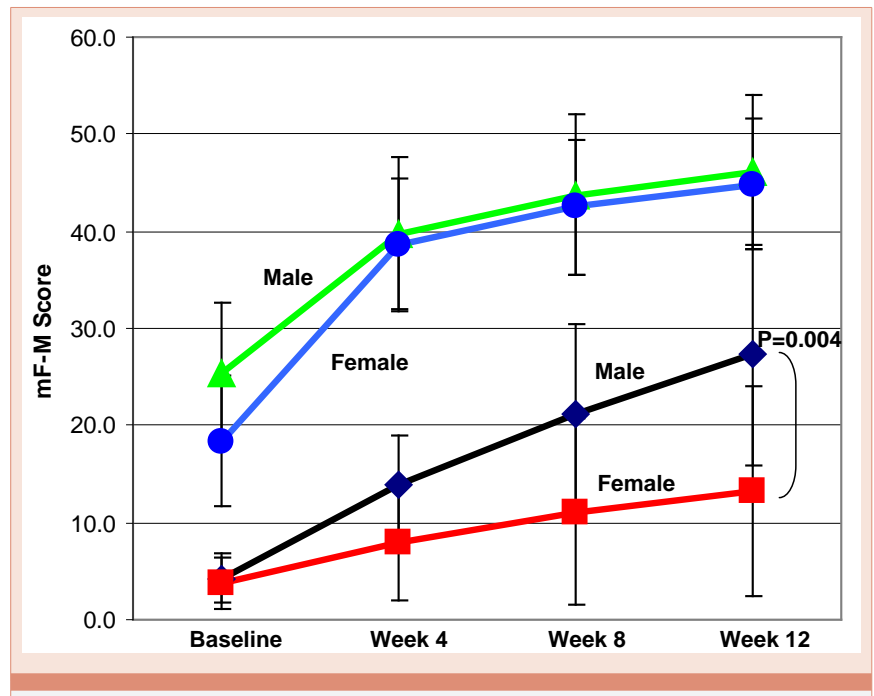

Figure 1: Recovery of motor control over 12 weeks of upper extremity rehabilitation.

Note: The lower two lines and the upper two lines represent patients with poor and good prognoses respectively
Yet on the functional tests $12 \%$ of males and $14 \%$ of females gain the ability to transfer blocks at a rate less than $20 \%$ below the non-paretic hand. Similarly, the time to complete the J-T test in no more than $30 \%$ over the non-paretic hand was achieved by $21 \%$ and $14 \%$ of males and females respectively. These data could not be tested for statistical significance due to the very small number of patients, however, it appears to suggest both females and males have similar chance for very good prognosis of recovering upper extremity function if their motor loss following stroke was not severe [24,25].

In contrast, the retrospective data presented herein suggest that women who had severe loss of motor control and functional ability are less likely to regain the same control as men after 12 weeks of intervention. This is despite receiving the same amount and dose of task-specific training with or without FES. These findings seem to parallel the findings of Wolf et al. [3,9] who focused on patients with less severe loss of motor control but only used one timed test to compare gender performance. Their team of investigators used the constraint-induced movement therapy, an intensive 2-week exercise program and measured as outcome, the time to complete the Wolf Motor Function Test. They reported that females were significantly slower than males. The gender factor was also the focus of Fukuda and colleagues' [5] study of 997 Japanese people who had suffered a stroke. The investigators also reported that women showed significantly worse locomotor function than men at both 1 and 5 years. In addition, females showed a poorer survival ratio after stroke. Taken together, these data support the general statement that stroke affects the physical performance of women more than men.

Why stroke has greater adverse impact on the motor system of women has not been directly addressed in the literature. Women may have more untreated pre-stroke co-morbidities, areless likely to receive antiplatelet agents within the first 48 hours after admission, suffer more frequently from atrial fibrillation, have higher systolic blood pressure, and a higher proportion of women remain unconscious or drowsy longer than men. In addition, a greater percentage of females may suffer from a total anterior circulation syndrome; a marker of less favorable prognosis $[2,4]$. The more pronounced motor and functional loss and slower rate of recovery, implies that women may require longer periods of physical intervention. The data of this study as well as prior publications provide preliminary evidence that most patients of both genders who started task-specific training shortly after stroke onset demonstrated a trend of improvement at study's end point (week 12) compared to week 4 or 8 [14,21].

One confounding factor in this secondary data analysis was the random assignment to task-specific or to task-specific + FES training. As a result of random assignment, the group of patients with poor prognosis only had 4 of 12 females versus 7 of 13 males trained with FES. The clearly smaller number of women trained with FES, may conceivably explain the slower progress of upper extremity recovery compared to men of similar prognosis. In fact, among currently offered rehabilitation interventions for upper extremity shortly after stroke, a growing number of publications support the added value of FES to task-specific training [14,15,21,26-30]. In addition, the added value of FES to augment further improvement of hand function has 
been demonstrated in patients with long standing chronic paresis of the upper extremity $[10,16,31]$. It may be plausible to project that by continuing the FES and task-specific training longer than 12 weeks, female subjects may continue to recover and minimize the motor control and functional RD to match those of males.

Inherent shortcomings of the study were the small sample size and uneven distribution of females and males in the two training groups. Similarly, potential comorbidities such as diabetes mellitus, prior cardio-pulmonary disease, or depression were not recorded. Moreover, limiting the training to only 12 weeks may not have been sufficient to maximize the recovery of upper extremity function particularly of female and less so of male with slower recovery rate. A future study design should consider both gender and severity of motor loss as primary factors, adequate sample size, and much longer training period particularly of patient with poor prognosis.

\section{Conclusions}

Loss of motor control and functional ability of the upper extremity is both clinically and statistically more evident in females following first-time ischemic stroke. Whereas the recovery rate associated with task-specific rehabilitation with or without FES appears similar in males and females whose loss is less severe (mF-M score 11-33), the deficits of motor control and hand function of females with severe initial loss (mF-M score 0-10) remain significantly higher than the deficits of males.

\section{References}

1. Jorgensen HS, Nakayama H, Reith J, Raaschou HO, Olsen TS (1997) Stroke recurrence: predictors, severity, and prognosis. The Copenhagen Stroke Study. Neurology 48: 891-895.

2. Niewada M, Kobayashi A, Sandercock PA, Kaminski B, Czlonkowska A (2005) Influence of gender on baseline features and clinical outcomes among 17,370 patients with confirmed ischaemic stroke in the international stroke trial. Neuroepidemiology 24: 123-128.

3. Wolf SL, Newton H, Maddy D, Blanton S, Zhang Q, Winstein CJ, et al. (2007) The Excite Trial: relationship of intensity of constraint induced movement therapy to improvement in the wolf motor function test. Restor Neurol Neurosci 25: 549-562.

4. Arrich J, Mullner M, Lalouschek W, Greisenegger S, Crevenna R, Herkner H (2008) Influence of socioeconomic status and gender on stroke treatment and diagnostics. Stroke 39: 2066-2072.

5. Fukuda M, Kanda T, Kamide N, Akutsu T, Sakai F (2009) Gender differences in long-term functional outcome after first-ever ischemic stroke. Intern Med 48: $967-973$

6. Kwakkel G, Kollen BJ, van der Grond J, Prevo AJ (2003) Probability of regaining dexterity in the flaccid upper limb: impact of severity of paresis and time since onset in acute stroke. Stroke 34: 2181-2186.

7. Cauraugh JH, Summers JJ (2005) Neural plasticity and bilateral movements: A rehabilitation approach for chronic stroke. Prog Neurobiol 75: 309-320.

8. McCombe Waller S, Whitall J (2008) Bilateral arm training: why and who benefits? NeuroRehabilitation 23: 29-41

9. Wolf SL, Thompson PA, Morris DM, Rose DK, Winstein CJ, Taub E, et al (2005) The EXCITE trial: attributes of the Wolf Motor Function Test in patients with subacute stroke. Neurorehabil Neural Repair 19: 194-205.

10. Alon G, Sunnerhagen KS, Geurts AC, Ohry A (2003) A home-based, selfadministered stimulation program to improve selected hand functions of chronic stroke. NeuroRehabilitation 18: 215-225

11. Daly JJ, Hogan N, Perepezko EM, Krebs HI, Rogers JM, Goyal KS, et al. (2005) Response to upper-limb robotics and functional neuromuscular stimulation following stroke. J Rehabil Res Dev 42: 723-736.

12. Hummel F, Celnik P, Giraux P, Floel A, Wu WH, Gerloff C, et al. (2005) Effects of non-invasive cortical stimulation on skilled motor function in chronic stroke. Brain 128: 490-499.

13. Lang CE, Beebe JA (2007) Relating movement control at 9 upper extremity segments to loss of hand function in people with chronic hemiparesis. Neurorehabil Neural Repair 21: 279-291.

14. Alon G, Levitt AF, McCarthy PA (2007) Functional electrical stimulation enhancement of upper extremity functional recovery during stroke rehabilitation: a pilot study. Neurorehabil Neural Repair 21: 207-215.

15. Chan MK, Tong RK, Chung KY (2009) Bilateral upper limb training with functional electric stimulation in patients with chronic stroke. Neurorehabil Neural Repair 23: 357-365.

16. Kimberley TJ, Lewis SM, Auerbach EJ, Dorsey LL, Lojovich JM, et al. (2004) Electrical stimulation driving functional improvements and cortical changes in subjects with stroke. Exp Brain Res 154: 450-460.

17. Mathiowetz V, Volland G, Kashman N, Weber K (1985) Adult norms for the Box and Block Test of manual dexterity. Am J Occup Ther 39: 386-391.

18. Jebsen RH, Taylor N, Trieschmann RB, Trotter MJ, Howard LA (1969) An objective and standardized test of hand function. Arch Phys Med Rehabil 50 311-319.

19. Wolf SL, Catlin PA, Ellis M, Archer AL, Morgan B, et al. (2001) Assessing Wolf motor function test as outcome measure for research in patients after stroke. Stroke 32: 1635-1639.

20. Woodbury ML, Velozo CA, Richards LG, Duncan PW, Studenski S, et al. (2007) Dimensionality and construct validity of the Fugl-Meyer Assessment of the upper extremity. Arch Phys Med Rehabil 88: 715-723.

21. Alon G, Levitt AF, McCarthy PA (2008) Functional electrical stimulation (FES) may modify the poor prognosis of stroke survivors with severe motor loss of the upper extremity: a preliminary study. Am J Phys Med Rehabil 87: 627636.

22. Ng YS, Stein J, Ning M, Black-Schaffer RM (2007) Comparison of clinical characteristics and functional outcomes of ischemic stroke in different vascular territories. Stroke 38: 2309-2314.

23. Alon G (2009) Defining and measuring residual deficits of the upper extremity following stroke: a new perspective. Top Stroke Rehabil 16: 167-176.

24. Hsieh YW, Lin KC, Wu CY, Lien HY, Chen JL, et al. (2014) Predicting clinically significant changes in motor and functional outcomes after robotassisted stroke rehabilitation. Arch Phys Med Rehabil 95: 316-321.

25. Houwink A, Nijland RH, Geurts AC, Kwakkel G (2013) Functional recovery of the paretic upper limb after stroke: who regains hand capacity? Arch Phys Med Rehabil 94: 839-844

26. Popovic DB, Popovic MB, Sinkjaer T, Stefanovic A, Schwirtlich L (2004) Therapy of paretic arm in hemiplegic subjects augmented with a neural prosthesis: a cross-over study. Can J Physiol Pharmacol 82: 749-756.

27. Berner YN, Lif Kimchi O, Spokoiny V, Finkeltov B (2004) The effect of electric stimulation treatment on the functional rehabilitation of acute geriatric patients with stroke--a preliminary study. Arch Gerontol Geriatr 39: 125-132.

28. Ring H, Rosenthal N (2005) Controlled study of neuroprosthetic functional electrical stimulation in sub-acute post-stroke rehabilitation. J Rehabil Med 37: 32-36.

29. Thrasher TA, Zivanovic V, Mcllroy W, Popovic MR (2008) Rehabilitation of reaching and grasping function in severe hemiplegic patients using functional electrical stimulation therapy. Neurorehabil Neural Repair 22: 706-714. 
30. Mangold S, Schuster C, Keller T, Zimmermann-Schlatter A, Ettlin T (2009) Motor training of upper extremity with functional electrical stimulation in early stroke rehabilitation. Neurorehabil Neural Repair 23: 184-190.
31. Santos M, Zahner LH, McKiernan BJ, Mahnken JD, Quaney B (2006) Neuromuscular electrical stimulation improves severe hand dysfunction for individuals with chronic stroke: a pilot study. J Neurol Phys Ther 30: 175-183.

Copyright: (c) 2014 Alon G. This is an open-access article distributed under the terms of the Creative Commons Attribution License, which permits unrestricted use, distribution, and reproduction in any medium, provided the original author and source are credited. 